\title{
CONDUTIVIDADE E DIFUSIVIDADE TÉRMICA DO FIGO (Ficus carica L.) "ROXO DE VALINHOS"
}

\section{SAUL D. SARRIA ${ }^{1}$, SYLVIO L. HONÓRIO}

RESUMO: O resfriamento e/ou congelamento de produtos hortícolas, depois da colheita, é realizado com o objetivo de retirar o calor desses produtos, permitindo-lhes, em função disso, um tempo maior de conservação. Portanto, o conhecimento das propriedades físicas que envolvem transferência de calor do figo "Roxo de Valinhos" é útil para o cálculo de projetos e a análise de sistemas de engenharia de alimentos em geral, assim como para o emprego em equações de modelos matemáticos termodinâmicos. Neste trabalho, foram determinadas, experimentalmente, a condutividade e a difusividade térmica do figo inteiro no estádio rami e, a partir desses valores, foi determinado o calor específico. Foi utilizado o método transiente da Fonte Linear de Calor. Foi introduzida nas frutas uma sonda que contém resistência elétrica e termopares. Para manter constante a temperatura da fruta, montou-se um sistema de resfriamento a água. Encontrou-se que o figo rami apresentou um valor de condutividade térmica de $0,52 \mathrm{~W} \mathrm{~m}^{-1}{ }^{\circ} \mathrm{C}$, difusividade térmica de $1,56 \times 10^{-7} \mathrm{~m}^{2} \mathrm{~s}^{-1}$, massa específica do figo de $815,6 \mathrm{~kg} \mathrm{~m}^{-3}$ e calor específico de $4,07 \mathrm{~kJ} \mathrm{~kg}^{-1}{ }^{\circ} \mathrm{C}$.

PALAVRAS-CHAVE: figo inteiro, transferência de calor, calor específico.

\section{CONDUCTIVITY AND THERMAL DIFFUSIVITY OF FIG FRUIT (Ficus carica L.) "ROXO DE VALINHOS"}

SUMMARY: The post harvest cooling and/or freezing processes for horticultural products have been carried out with the objective of removing the heat from these products, allowing them a bigger period of conservation. Therefore, the knowledge of the physical properties that involve heat transference in the fig fruit "Roxo de Valinhos" is useful for calculating projects and systems of food engineering in general, as well as, for using in equations of thermodynamic mathematical models. The values of conductivity and thermal diffusivity of the whole fig fruit-rami index were determined, and from these values it was determined the value of the specific heat. For these determination it was used the transient method of the Line Heat Source. The results shown that the fig fruit has a thermal conductivity of $0.52 \mathrm{~W} \mathrm{~m}^{-1}{ }^{\circ} \mathrm{C}$, thermal diffusivity of $1.56 \times 10^{-7} \mathrm{~m}^{2} \mathrm{~s}^{-1}$, pulp density of $815.6 \mathrm{~kg} \mathrm{~m}^{-3}$ and specific heat of $4.07 \mathrm{~kJ} \mathrm{~kg}^{-1}{ }^{\circ} \mathrm{C}$.

KEYWORDS: fig fruit, heat transfer, specific heat.

\section{INTRODUÇÃO}

O resfriamento e/ou congelamento de frutos e hortaliças, depois da colheita, é realizado com o objetivo de conservar suas características organolépticas e evitar o desenvolvimento de microrganismos indesejáveis. Com isso, obtém-se o tempo adequado de conservação traduzido em vida de prateleira mais longa.

\footnotetext{
${ }^{1}$ M.Sc. em Engenharia Agrícola, Estudante de Doutorado em Engenharia Agrícola, FEAGRI/UNICAMP, Caixa Postal 6011, Campinas SP, Fax: (0XX19) 3788.1010, e-mail: saul@agr.unicamp.br, Bolsista da FAPESP.

${ }^{2}$ Prof. Dr., FEAGRI/UNICAMP, Caixa Postal 6011, Campinas - SP, e-mail: honório@agr.unicamp.br

Recebido pelo Conselho Editorial em: 13-2-2002

Aprovado pelo Conselho Editorial em: 22-9-2003
} 
A falta de valores tabulados das propriedades térmicas do figo "Roxo de Valinhos" tem restringido o emprego de equações, em modelos matemáticos, para o cálculo das propriedades termodinâmicas.

ASHRAE (1993) descreve as equações de Siebel para o cálculo do calor específico dos materiais biológicos com base no conteúdo de água, para temperaturas acima de $0{ }^{\circ} \mathrm{C}$.

$$
\mathrm{C}_{\mathrm{p}}=0,0335 \mathrm{X}_{\mathrm{w}}+0,837
$$

em que,

$\mathrm{C}_{\mathrm{p}}$ - calor específico, $\mathrm{kJ} \mathrm{kg}^{-1}{ }^{\circ} \mathrm{C}$, e

$\mathrm{X}_{\mathrm{w}}$ - conteúdo de água, $\%$. eq.(2).

SWEAT (1974) determinou a condutividade térmica de vários frutos e hortaliças expressa pela

$$
\mathrm{k}=0,00493 \mathrm{X}_{\mathrm{w}}+0,148
$$

em que,

$\mathrm{k}$ - condutividade térmica, $\mathrm{W} \mathrm{m}^{-1}{ }^{\circ} \mathrm{C}$.

CHOI \& OKOS (1986) desenvolveram um modelo matemático [eq.(3)] para o cálculo da condutividade térmica da água $\left(\mathrm{k}_{\mathrm{w}}\right)$ em função da sua temperatura $(T)$.

$$
\mathrm{k}_{\mathrm{w}}=0,57109+1,7625 \times 10^{-3} \mathrm{~T}-6,703 \times 10^{-6} \mathrm{~T}^{2}
$$

em que, $\mathrm{T}$ é expresso em ${ }^{\circ} \mathrm{C}$.

SWEAT (1974) relatou para pêssego com $88,5 \%$ de água, à temperatura de $28^{\circ} \mathrm{C}$, um valor de $\mathrm{k}$ igual a $0,581 \mathrm{~W} \mathrm{~m}^{-1}{ }^{\circ} \mathrm{C}$ e para pêra com $86,8 \%$ de água, à temperatura de $28^{\circ} \mathrm{C}$, um valor de $\mathrm{k}=0,595 \mathrm{~W} \mathrm{~m}^{-1}{ }^{\circ} \mathrm{C}$.

A difusividade térmica é mais utilizada em situações em que a transferência de calor ocorre em regime transiente e definida matematicamente pela eq.(4).

$$
\alpha=\frac{\mathrm{k}}{\rho \mathrm{C}_{\mathrm{p}}}
$$

em que,

$\alpha$ - difusividade térmica, $\mathrm{m}^{2} \mathrm{~s}^{-1}$;

$\rho$ - massa específica, $\mathrm{kg} \mathrm{m}^{-3}$, e

$\mathrm{C}_{\mathrm{p}}$ - calor específico, $\mathrm{kJ} \mathrm{kg}^{-1}{ }^{\circ} \mathrm{C}$.

De acordo com MOHSENIN (1980), o figo in natura inteiro (cultivar não identificada), com conteúdo de água de $78 \%$, apresenta um calor específico de $3,43 \mathrm{~kJ} \mathrm{~kg}^{-1}{ }^{\circ} \mathrm{C}$, acima do ponto de congelamento.

RIEDEL (1969) verificou que a difusividade térmica de alimentos com conteúdo de água maior que $40 \%$ é fortemente dependente do conteúdo de água $\left(\mathrm{X}_{\mathrm{w}}\right)$. A expressão que representa essa dependência é a seguinte:

$$
\alpha=0,08810^{-6}+\left(\alpha_{\mathrm{w}}-0,08810^{-6}\right) \frac{\mathrm{X}_{\mathrm{w}}}{100}
$$

em que, $\alpha_{\mathrm{w}}$ é a difusividade térmica da água expressa em $\mathrm{m}^{2} \mathrm{~s}^{-1}$. 
HAYAKAWA \& SUCCAR (1983) determinaram a difusividade térmica de tomates frescos obtendo valores da ordem de $1,80 \times 10^{-7} \mathrm{~m}^{2} \mathrm{~s}^{-1}$ à temperatura de $43{ }^{\circ} \mathrm{C}$.

Segundo MURAKAMI et al. (1996), em alimentos com alto conteúdo de água (>70\%), o valor da difusividade térmica de $1,4 \times 10^{-7} \mathrm{~m}^{2} \mathrm{~s}^{-1}$ é aceitável, porém recomenda-se fazer a respectiva correção para o material particular a ser avaliado.

Um dos métodos utilizados para a determinação da condutividade térmica é o método da fonte linear de calor. No método de estado variável ou transiente, para determinar a condutividade térmica, utiliza-se de uma fonte linear de calor para gerar um fluxo de calor constante na amostra, a qual deverá estar inicialmente em equilíbrio térmico (MOHSENIN, 1980). Esse método apresenta algumas dificuldades como: medição da temperatura, localização dos termopares, transferência de calor convectiva em medições de propriedades térmicas de materiais granulares e controle da temperatura na amostra.

INGERSOLL et al. (1954), NIX et al. (1967) e MOHSENIN (1980) demonstraram a utilização do método da fonte linear de calor, considerando um ponto no material de condutividade térmica $(\mathrm{k})$ que recebe uma fonte linear de calor (q), que provoca a elevação de temperatura de $T_{1}$ para $T_{2}$, no intervalo de tempos $t_{1}$ a $t_{2}$ e pode ser representada pela eq.(6).

$$
\mathrm{T}_{2}-\mathrm{T}_{1}=\frac{\mathrm{q}}{4 \pi \mathrm{k}} \ln \left(\frac{\mathrm{t}_{2}}{\mathrm{t}_{1}}\right)
$$

em que,

$\mathrm{k}$ - condutividade térmica experimental, $\mathrm{W} \mathrm{m}^{-1}{ }^{\circ} \mathrm{C}$, e

$\mathrm{q}$ - calor proporcionado à amostra, $\mathrm{W} \mathrm{m}^{-1}$.

A determinação da difusividade térmica $\alpha$, utilizando o método da fonte linear, é possível. NIX et al. (1967) descreveram a seguinte série:

$$
\begin{aligned}
& \Delta \mathrm{T}=\frac{\mathrm{q}}{2 \pi \mathrm{k}}\left[\frac{-\mathrm{C}}{2}-\ln \beta+\frac{\beta^{2}}{2 \times 1 !}-\frac{\beta^{4}}{4 \times 2 !}+\frac{\beta^{6}}{6 \times 3 !}-\ldots\right] \\
& \beta=\frac{\mathrm{r}}{2 \sqrt{\alpha \mathrm{t}}}
\end{aligned}
$$

em que,

$\Delta \mathrm{T}$ - elevação de temperatura de $\mathrm{T}_{1}$ para $\mathrm{T}_{2},{ }^{\circ} \mathrm{C}$;

C - constante de Euler, 0,57721;

$\beta$ - parâmetro adimensional, e

$\alpha_{\mathrm{w}}$ - difusividade térmica da água, $\mathrm{m}^{2} \mathrm{~s}^{-1}$.

DINCER (1995) argumenta que o significado físico da difusividade térmica está associado à difusão de calor dentro do produto durante as mudanças da temperatura com o tempo. Um elevado valor da difusividade térmica significa uma rápida transferência do calor dentro do produto e pouco tempo para o calor sair do corpo. CHAU (2001) considera importante a determinação experimental dos valores da condutividade e da difusividade térmica para o cálculo do coeficiente convectivo de transferência de calor e a taxa de resfriamento durante o processo de resfriamento de produtos hortícolas.

Dessa forma, este trabalho foi desenvolvido com o objetivo de determinar, experimentalmente, a condutividade e a difusividade térmica do figo inteiro no estádio rami e, a partir desses valores, determinar seu calor específico. 


\section{MATERIAL E MÉTODOS}

Os testes para a determinação da condutividade e da difusividade térmica foram feitos no Laboratório de Medidas Físicas da Faculdade de Engenharia de Alimentos/UNICAMP. A determinação da massa específica da polpa dos frutos foi feita no Laboratório de Pós-Colheita de Frutas e Hortaliças na Faculdade de Engenharia Agrícola/UNICAMP.

\section{Amostras}

Os figos utilizados foram procedentes da região de Valinhos - SP, safra 2000, e apresentavam boa aparência, sendo classificados como tipo $8 \mathrm{em}$ estádio rami. Os frutos foram obtidos aleatoriamente de uma amostra de 30 frutos.

\section{Montagem experimental}

Para a determinação da condutividade e da difusividade térmica, foi utilizada uma sonda com duas agulhas. A agulha de maior diâmetro contém um fio de resistência elétrica e um termopar usado para medir as mudanças de temperatura para o cálculo da condutividade térmica e outra agulha de menor diâmetro onde se encontra um termopar usado para medir as mudanças de temperatura para o cálculo da difusividade térmica. O fio de resistência utilizado foi o wire-up, bitola 32, encapado com PVC. O comprimento da agulha era de $4 \mathrm{~cm}$, e o comprimento total do fio, em forma de U, dentro da agulha, era de $8 \mathrm{~cm}$. A distância entre as agulhas era de $4 \mathrm{~mm}$, que corresponde a $\mathrm{r}$ na eq.(8). Para estabilizar e manter a temperatura da amostra, montou-se um sistema de resfriamento a água, utilizando-se de um banho termostático. Dentro da cuba com água e com a ajuda de um suporte, foi colocado o fruto e, dentro do fruto, a respectiva sonda. Foram feitas quatro repetições, duas repetições introduzindo a sonda na direção longitudinal do fruto e outras duas introduzindo a sonda na direção radial ao fruto. Teve-se a precaução de colocar o extremo da sonda na polpa do fruto e não no espaço vazio interno do fruto para evitar falsas leituras. As temperaturas mantidas nos frutos, ou temperaturas iniciais, variaram entre 5 e $21^{\circ} \mathrm{C}$.

A montagem experimental é mostrada na Figura 1. Os termopares da sonda foram ligados a um registrador de temperatura de marca Digi-Sense Scannig Thermocouple Thermometer, fabricado pela Cole Parmer e este, por sua vez, ligado a um computador. O programa Scan-Log V1.5 foi utilizado para fazer a leitura dos dados de tempo (s) e temperatura $\left({ }^{\circ} \mathrm{C}\right)$. Para cada teste, os dados foram adquiridos a cada $4 \mathrm{~s}$, durante um intervalo de tempo de 20 minutos. Portanto, a cada $4 \mathrm{~s}$, foram registrados os valores da variação de temperatura com uma precisão de $\pm 0,1{ }^{\circ} \mathrm{C}$. Em média, foram obtidos 300 pontos em cada teste.

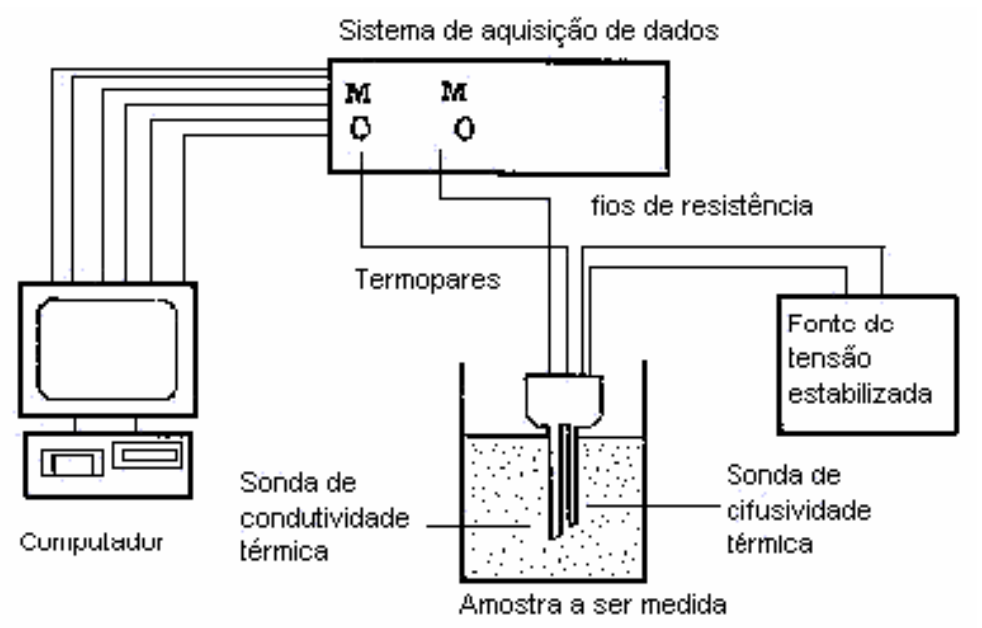

FIGURA 1. Esquema de montagem experimental para a aquisição dos dados de tempo e temperatura. 


\section{Calibração da sonda}

Na calibração da sonda, foi utilizada água destilada como substância de valor real conhecido e ágar, representando $2 \%$ em peso do total de cada amostra. Durante a calibração, foram feitas quatro repetições. Com a calibração da sonda, foi possível determinar o fator de correção (f) para a condutividade térmica e o fator (h) para a difusividade térmica, por meio das eqs. (9) e (10).

$$
\mathrm{f}=\frac{\mathrm{k}_{\text {teórico }}}{\mathrm{k}_{\text {exp erimental }}}
$$

em que, $\mathrm{k}_{\text {teórico }}$ corresponde aos valores calculados segundo a eq.(3).

$$
\mathrm{h}=\frac{\alpha_{\text {térico }}}{\alpha_{\text {experimental }}}
$$

Os valores de $\alpha_{\text {teórico }}$ foram adquiridos por meio de tabelas na literatura (CHOI \& OKOS, 1986). Nessa referência bibliográfica, a difusividade térmica da água, na faixa de temperatura de 5 a $21{ }^{\circ} \mathrm{C}$, corresponde a, aproximadamente, $1,45 \times 10^{-7} \mathrm{~m}^{2} \mathrm{~s}^{-1}$. Para obter os valores corrigidos da condutividade e difusividade térmica dos frutos, multiplicaram-se os valores experimentais pelos valores de $\mathrm{f}$ e $\mathrm{h}$, respectivamente.

\section{Determinação da condutividade térmica}

A sonda foi introduzida no fruto e aguardou-se um tempo para estabilizar a temperatura desejada. A variação da temperatura do fruto, ao longo do tempo, foi registrada obtendo-se a curva $\ln (\mathrm{t})$ em função de $\mathrm{T}$. A região que melhor representou o comportamento linear, com base no maior coeficiente de determinação $\left(R^{2}\right)$, foi escolhida para o cálculo. A corrente elétrica foi de 3,3 A, no fio de resistência de $0,5 \Omega \mathrm{m}^{-1}$, gerando uma quantidade de calor no interior da amostra de $4,59 \mathrm{~W} \mathrm{~m}^{-1}$. A condutividade térmica $(\mathrm{k})$ foi determinada por meio da eq.(6).

\section{Determinação da difusividade térmica}

A partir dos resultados obtidos da condutividade térmica dos frutos e utilizando a eq.(7), foi possível calcular a difusividade térmica. Para isso, a eq.(7) foi igualada a zero, e substituindo os valores de cada diferença acumulada de temperatura, foram calculados os valores de $\beta$, utilizando o programa de análise numérica, Mathematics for Windows, Versão 2.2.3, por meio do método da secante, para encontrar a raiz da equação. Para cada diferença de temperatura, existe um tempo gasto durante o aquecimento da amostra que corresponde a t. Procurou-se utilizar a mesma faixa de tempo para a seleção dos pares de dados em cada teste (o tempo acumulado correspondeu a, aproximadamente, $300 \mathrm{~s}$ ). Foram substituídos na eq.(8) os valores de $\beta$ e de t, para determinar $\alpha$, que corresponde à difusividade térmica experimental. Esse procedimento foi feito tanto na calibração da sonda quanto na determinação propriamente dita dos valores das propriedades das frutas de figo.

\section{Determinação da massa específica}

A massa específica foi determinada a partir da relação entre a massa e o volume do produto. A massa de cada fruta foi determinada em uma balança analítica e o seu volume, aplicando o princípio de Arquimedes. Conhecida a massa e o volume de água deslocado pela fruta, determinou-se a massa específica $(\rho)$ pela eq.(11). Foram utilizadas vinte frutas para essa determinação.

$$
\rho=\frac{\mathrm{m}}{\mathrm{V}}
$$

em que, 
$\mathrm{V}$ - volume da fruta, $\mathrm{m}^{3}$;

$\mathrm{m}$ - massa da fruta, $\mathrm{kg}$, e

$\rho$ - massa especifica da fruta, $\mathrm{kg} \mathrm{m}^{-3}$.

\section{Determinação do calor específico}

Conhecendo-se os valores experimentais da condutividade térmica, da difusividade térmica e da massa específica, o calor específico pode ser determinado matematicamente pela eq.(4).

\section{Análise dos dados}

Para o tratamento estatístico dos dados, foi aplicada a estatística descritiva simples, utilizando a média, o desvio-padrão (DP) e o coeficiente de variação (CV). Com os dados experimentais para o cálculo da condutividade térmica, foram feitos ajustes de modelos por meio de regressão linear.

\section{RESULTADOS E DISCUSSÃO}

\section{Condutividade térmica}

Na Tabela 1, observam-se os resultados da condutividade térmica da calibração da amostra (água-ágar), obtendo-se o fator médio de correção (f). Os coeficientes de determinação ( ${ }^{2}$ ), que explicam o modelo linear, foram de 0,99 .

TABELA 1. Calibração da sonda (água-ágar) para determinar a condutividade térmica (k).

\begin{tabular}{ccccccc}
\hline Amostra & $\begin{array}{c}\text { Temperatura da } \\
\text { Amostra }\left({ }^{\circ} \mathrm{C}\right)\end{array}$ & $\mathrm{b}$ & $\mathrm{R}^{2}$ & $\begin{array}{c}\mathrm{k} \\
\left(\mathrm{W} \mathrm{m}^{-1}{ }^{\circ} \mathrm{C}\right)\end{array}$ & $\begin{array}{c}\mathrm{k}_{\text {água térico }}\left(\mathrm{W} \mathrm{m}^{-1}{ }^{\circ} \mathrm{C}\right) \\
\text { Fator de Correção }\end{array}$ & $\begin{array}{c}\mathrm{f} \\
\text { 1 }\end{array}$ \\
3 & 7 & 0,6203 & 0,99 & 0,2266 & 0,576 & 2,5434 \\
2 & 9 & 0,5702 & 0,99 & 0,2083 & 0,583 & 2,7994 \\
3 & 25 & 0,8779 & 0,99 & 0,3207 & 0,586 & 1,8285 \\
4 & 0,5920 & 0,99 & 0,2163 & 0,611 & 2,8252 \\
\hline Média & & & & & 2,4991 \\
\hline
\end{tabular}

$\mathrm{b}$ - inclinação ou pendente da linha reta $\ln (\mathrm{t}) / \Delta \mathrm{T} ; \mathrm{R}^{2}$ - coeficiente de determinação do ajuste.

Na Tabela 2, observam-se os valores experimentais corrigidos da condutividade térmica do fruto de figo rami. Os valores corrigidos resultaram da multiplicação dos valores experimentais pelo valor médio do fator de correção. A média final da condutividade térmica do figo foi de, aproximadamente, $0,52 \mathrm{~W} \mathrm{~m}^{-1}{ }^{\circ} \mathrm{C}$, o coeficiente de variação foi de, aproximadamente, $9 \%$, o que mostra pouca dispersão dos dados com respeito à média.

TABELA 2. Valores da condutividade térmica (k) do figo rami.

\begin{tabular}{|c|c|c|c|c|c|}
\hline Amostra & $\begin{array}{c}\text { Temperatura da } \\
\text { Amostra }\left({ }^{\circ} \mathrm{C}\right)\end{array}$ & b & $\mathrm{R}^{2}$ & $\begin{array}{l}\frac{\mathrm{k}_{\text {experimental }}}{\mathrm{W} \mathrm{m}{ }^{-1}{ }^{\circ} \mathrm{C}} \\
\end{array}$ & $\begin{array}{l}\mathrm{k}_{\text {corrigido }} \\
\mathrm{W} \mathrm{m}^{-1}{ }^{\circ} \mathrm{C}\end{array}$ \\
\hline 1 & 5 & 0,5373 & 0,94 & 0,1963 & 0,4905 \\
\hline 2 & 11 & 0,5089 & 0,95 & 0,1859 & 0,4646 \\
\hline 3 & 15 & 0,6126 & 0,94 & 0,2238 & 0,5593 \\
\hline 4 & 21 & 0,6108 & 0,93 & 0,2231 & 0,5576 \\
\hline Média & & & & & 0,5180 \\
\hline DP & & & & & 0,0479 \\
\hline C.V. $(\%)$ & & & & & 9,2 \\
\hline
\end{tabular}

$\mathrm{b}$ - inclinação ou pendente da linha reta $\ln (\mathrm{t}) / \Delta \mathrm{T} ; \mathrm{R}^{2}$ - coeficiente de determinação do ajuste. 
Segundo DUSSÁN \& HONÓRIO (2000), o figo "Roxo de Valinhos", no ponto de colheita rami, possui $84,3 \%$ de conteúdo de água. Substituindo esse valor na eq.(2), tem-se o valor da condutividade térmica de $0,56 \mathrm{~W} \mathrm{~m}^{-1}{ }^{\circ} \mathrm{C}$. SWEAT (1974) reporta para pêssego uma condutividade térmica de $0,581 \mathrm{~W} \mathrm{~m}^{-1}{ }^{\circ} \mathrm{C}$ e para pêra, de $0,595 \mathrm{~W} \mathrm{~m}^{-1}{ }^{\circ} \mathrm{C}$. Portanto, observa-se que o valor experimental encontrado é próximo dos valores estimados por meio dos modelos propostos na literatura e reportados para outros frutos carnosos.

\section{Difusividade térmica}

Na Tabela 3, observam-se os valores da difusividade térmica das amostras água-ágar e seus respectivos fatores de correção (h).

TABELA 3. Calibração da sonda (água-agar) para determinar a difusividade térmica $(\alpha)$.

\begin{tabular}{cccccc}
\hline Amostra & Tempo $(\mathrm{s})$ & $\Delta \mathrm{T}\left({ }^{\circ} \mathrm{C}\right)$ & $\beta$ & $\alpha\left(\times 10^{-7} \mathrm{~m}^{2} \mathrm{~s}^{-1}\right)$ & Fator de Correção $(\mathrm{h})$ \\
\hline 1 & 309 & 1,7 & 0,195 & 3,4 & 0,43 \\
2 & 304 & 2,9 & 0,206 & 3,1 & 0,47 \\
3 & 308 & 1,4 & 0,244 & 2,2 & 0,66 \\
4 & 300 & 1,7 & 0,195 & 3,5 & 0,41 \\
\hline Média & \multicolumn{5}{c}{}
\end{tabular}

$\beta$ - parâmetro adimensional, determinado por meio da eq.(7); $\alpha$ - difusividade térmica experimental.

Os valores corrigidos da difusividade térmica do figo rami encontram-se na Tabela 4. O coeficiente de variação de $42,9 \%$ indica que os dados apresentaram grande variação em relação ao valor médio, variação que se deveu, em parte, à dificuldade experimental, principalmente no controle da temperatura da amostra.

TABELA 4. Valores da difusividade térmica $(\alpha)$ do figo rami.

\begin{tabular}{cccccc}
\hline Amostra & Tempo $(\mathrm{s})$ & $\Delta \mathrm{T}\left({ }^{\circ} \mathrm{C}\right)$ & $\beta$ & $\alpha\left({\left.\mathrm{x} 10^{-7} \mathrm{~m}^{2} \mathrm{~s}^{-1}\right)} \alpha\right.$ corrigido $\left({\left.\mathrm{x} 10^{-7} \mathrm{~m}^{2} \mathrm{~s}^{-1}\right)}^{-1,73}\right.$ \\
2 & 388 & 2,1 & 0,171 & 3,53 & 1,30 \\
3 & 284 & 1,7 & 0,230 & 2,66 & 0,80 \\
4 & 396 & 1,6 & 0,248 & 1,64 & 2,39 \\
\hline Média & 400 & 2,4 & 0,143 & 4,88 & 1,56 \\
DP & & & & & 0,67 \\
C.V. $(\%)$ & & & & & 42,9 \\
\hline
\end{tabular}

$\beta$ - parâmetro adimensional, determinado por meio da eq.(7); $\alpha$ - difusividade térmica experimental.

Considerando-se o coeficiente de variação $(\mathrm{CV})$ de $42,9 \%$ do $\alpha_{\text {corrigido, }}$ pode-se ter: $\alpha_{\text {médio }}=$ $1,56 \times 10^{-7} \mathrm{~m}^{2} \mathrm{~s}^{-1} ; \alpha_{\text {alto }}=42,9 \%$ acima do valor de $\alpha_{\text {médio, }}$ e $\alpha_{\text {baixo }}=42,9 \%$ abaixo do valor de $\alpha_{\text {médio. }}$. Determinou-se o calor específico (Cp), para cada $\alpha$, com base na eq.(4), assumindo-se a condutividade térmica $(\mathrm{k})=0,52 \mathrm{~W} \mathrm{~m}^{-1}{ }^{\circ} \mathrm{C}$ e $\rho=815,6 \mathrm{~kg} \mathrm{~m}^{-3}$ (Tabela 5). Por meio da eq.(12), obteve-se o calor sensível $\left(\mathrm{q}_{\mathrm{s}}\right)$ gerado pela fruta, assumindo-se uma massa $(\mathrm{m})$ de $100 \mathrm{~kg}$ e uma diferença de temperatura no resfriamento $(\Delta \mathrm{T})$ de $18^{\circ} \mathrm{C}$.

$$
\mathrm{q}_{\mathrm{s}}=\mathrm{m} \mathrm{C}_{\mathrm{p}} \Delta \mathrm{T}
$$


TABELA 5. Valores de calor sensível com diferentes calores específicos.

\begin{tabular}{cccr}
\hline$\alpha$ & $\alpha\left(\times 10^{-7} \mathrm{~m}^{2} \mathrm{~s}^{-1}\right)$ & $\mathrm{Cp}\left(\mathrm{kJ} \mathrm{kg}^{-1}{ }^{\circ} \mathrm{C}\right)$ & $\mathrm{q}_{\mathrm{s}}(\mathrm{kJ})$ \\
\hline Alto & 2,20 & 2,90 & $5.220,0$ \\
Médio & 1,56 & 4,09 & $7.362,0$ \\
Baixo & 0,92 & 6,93 & $12.474,0$ \\
\hline
\end{tabular}

$\alpha$ - difusividade térmica; $\mathrm{Cp}$ - calor especifico; $\mathrm{q}_{\mathrm{s}}$ - calor sensível.

Verificou-se que a diferença entre o calor sensível para $\alpha_{\text {médio }}$ e $\alpha_{\text {alto }}$ foi de $2.142 \mathrm{~kJ}$ e entre $\alpha_{\text {baixo }}$ e $\alpha_{\text {médio }}$ de $5.112 \mathrm{~kJ}$. Para representar melhor essas diferenças, converteram-se esses valores de calor produzido pelo figo em potência e, considerando-se o tempo de uma hora, $2.142 \mathrm{~kJ}$ equivale a $0,595 \mathrm{kWh}$ e $5.112 \mathrm{~kJ}$ a $1,420 \mathrm{kWh}$. Isso indica que, embora os dados da difusividade térmica tenham apresentado um valor de CV alto, na prática, essas diferenças transformadas em calor sensível não são significativas. Com base na eq.(5) e para o conteúdo de água da polpa de figo de $84,3 \%$, obteve-se uma difusividade de $1,36 \times 10^{-7} \mathrm{~m}^{2} \mathrm{~s}^{-1}$, considerando-se o valor da difusividade térmica da água de $1,45 \times 10^{-7} \mathrm{~m}^{2} \mathrm{~s}^{-1}$. HAYAKAWA \& SUCCAR (1983) encontraram, para tomates frescos, um valor de difusividade térmica de $1,80 \times 10^{-7} \mathrm{~m}^{2} \mathrm{~s}^{-1}$, valor esse maior que o encontrado para o figo, devido ao fato de o tomate apresentar, em média, conteúdo de água maior que $90 \%$ e massa específica diferente. $\mathrm{Na}$ eq.(5), observa-se que, para maior conteúdo de água, a difusividade térmica dos alimentos é maior.

\section{Massa específica}

Na Tabela 6, são apresentados os valores experimentais da massa específica do figo rami, valor necessário para o cálculo do calor específico do material.

TABELA 6. Valores da massa específica do figo rami.

\begin{tabular}{cccc}
\hline Fruto & Massa $(\mathrm{g})$ & Volume $\left(\mathrm{m}^{3}\right)$ & Massa Específica $\left(\mathrm{kg} \mathrm{m}^{-3}\right)$ \\
\hline 1 & 63,6 & 0,00009 & 703,1 \\
2 & 69,5 & 0,00009 & 814,5 \\
3 & 96,9 & 0,00013 & 749,1 \\
4 & 80,1 & 0,00009 & 854,2 \\
5 & 76,3 & 0,00009 & 819,9 \\
6 & 105,6 & 0,00013 & 835,9 \\
7 & 65,1 & 0,00008 & 799,7 \\
8 & 70,3 & 0,00008 & 853,0 \\
9 & 100,7 & 0,00011 & 896,2 \\
10 & 72,1 & 0,00010 & 729,8 \\
11 & 75,1 & 0,00009 & 840,5 \\
12 & 60,6 & 0,00009 & 683,6 \\
13 & 81,7 & 0,00010 & 812,1 \\
14 & 80,1 & 0,00009 & 872,0 \\
15 & 87,5 & 0,00010 & 873,2 \\
16 & 73,3 & 0,00009 & 802,3 \\
17 & 78,1 & 0,00009 & 829,3 \\
18 & 82,5 & 0,00010 & 804,0 \\
19 & 82,0 & 0,00010 & 830,0 \\
20 & 84,2 & 0,00009 & 908,7 \\
\hline Média & & & 815,6 \\
DP & & & 60,0 \\
C.V.(\%) & & & 7,4 \\
\hline
\end{tabular}


Observa-se a homogeneidade da amostra (C.V. $=7,4 \%)$. O figo rami apresenta massa específica média de $815,6 \mathrm{~kg} \mathrm{~m}^{-3}$.

\section{Calor específico}

Substituindo os valores experimentais encontrados da condutividade térmica $\left(0,52 \mathrm{~W} \mathrm{~m}^{-1}{ }^{\circ} \mathrm{C}\right)$, difusividade térmica $\left(1,56 \times 10^{-7} \mathrm{~m}^{2} \mathrm{~s}^{-1}\right)$ e massa específica $\left(815,6 \mathrm{~kg} \mathrm{~m}^{-3}\right)$ na eq.(4), resulta o calor específico de $4,07 \mathrm{~kJ} \mathrm{~kg}^{-1}{ }^{\circ} \mathrm{C}$. Por meio da eq.(1), equação de Siebel, para figo rami com conteúdo de água de $84,3 \%$, obtém-se o calor específico de 3,66 $\mathrm{kJ} \mathrm{kg}^{-1}{ }^{\circ} \mathrm{C}$. Segundo MOHSENIN (1980), o figo in natura (cultivar não identificada) apresenta um calor específico de $3,43 \mathrm{~kJ} \mathrm{~kg}^{-1}{ }^{\circ} \mathrm{C}$ para um conteúdo de água de 78\%. Apesar de serem espécies distintas de figo, e o resultado da literatura conseguido não identificar a cultivar, pode-se afirmar que as variações dos valores do calor específico encontrados, para efeito de cálculo de calor sensível $(15,7 \%)$, são pouco significativas. Considerando-se uma mesma massa específica do produto, o valor do calor específico é relativo, pois, proporcionalmente a valores menores ou maiores da condutividade térmica e da difusividade térmica, ter-se-ia um mesmo valor de Cp [eq.(4)].

\section{CONCLUSÕES}

O figo em estádio rami "Roxo de Valinhos" apresenta condutividade térmica de $0,52 \mathrm{~W} \mathrm{~m}^{-1}{ }^{\circ} \mathrm{C}$, difusividade térmica de $1,56 \times 10^{-7} \mathrm{~m}^{2} \mathrm{~s}^{-1}$, calor específico aproximado de $4,07 \mathrm{~kJ} \mathrm{~kg}^{-1}{ }^{\circ} \mathrm{C}$ e massa específica de $815,6 \mathrm{~kg} \mathrm{~m}^{-3}$.

Os valores da difusividade térmica e do calor específico do figo cultivar Roxo de Valinhos, aqui encontrados, são maiores que os valores reportados para figo de cultivares diferentes.

Os valores relativamente altos da condutividade térmica e difusividade térmica do figo indicam certa vantagem no que diz respeito a processos de resfriamento ou aquecimento da fruta.

Para a metodologia utilizada, encontrou-se dificuldade no controle da temperatura da amostra durante as determinações.

\section{AGRADECIMENTOS}

Os autores agradecem à FAPESP (Fundo de Amparo à Pesquisa no Estado de São Paulo), pelo suporte financeiro a este trabalho.

\section{REFERÊNCIAS BIBLIOGRÁFICAS}

ASHRAE. Handbook of Fundamentals. Atlanta: American Society of Heating, Refrigerating and Air Conditioning Engineers, 1993.

CHAU, K.V. Mathematical modeling of post harvest processes. Campinas: Grupo de Tecnologia PósColheita. Universidade Estadual de Campinas. Faculdade de Engenharia Agrícola, 2001. (Apostila)

CHOI, Y.; OKOS, M.R. Effects of temperature and composition on the thermal properties of foods. In: LEMAUGUER, M.; JELEN, M. Food Engineering and Process Aplications. Elsevier Applied Science Publishers, 1986. p.93-101.

DINCER, I. Thermal diffusivities of geometrical objects subject to cooling. Applied Energy, London, v.51, p.111-8, 1995.

DUSSÁN, S.S.; HONÓRIO, S.L. Caracterização física do figo (Fícus carica L.) "Roxo de Valinhos" com diferentes graus de maturidade. In: CONGRESSO BRASILEIRO DE ENGENHARIA 
AGRÍCOLA, 29., 2000, Fortaleza. Anais... Fortaleza: Sociedade Brasileira de Engenharia Agrícola, 2000. 1 CD.

HAYAKAWA, K.; SUCCAR, J. A method for determining the apparent thermal diffusivity of sphere foods. Lebensm. Wiss. u. Tech, v.16, p.373-5, 1983.

INGERSOLL, L.R.; ZOBEL, J.; INGERSOLL, A.C. Heat conduction for engineering, geological, and other applications. University of Wisconsin Press, 1954. p 284-9.

MOHSENIN, N.N. Thermal properties of foods and agricultural materials. New York: Gordon and Breach, 1980. p.198-224.

MURAKAMI, E.G.; SWEAT, V.E.; SASTRY, S.K.; KOLBE, E.; HAYAKAWA, K.; DATTA, A. Recommended design parameters for thermal conductivity probes for non frozen food materials. Journal of Food Engineering, Kidlington, v.27 p.109-23, 1996.

NIX, G.H.; LOWERY, G.W.; VACHON, R.I.; TANGER, G.E. Direct determination of thermal diffusivity and conductivity with a refined line source technique. Progress in aeronautics and astronautics. Thermophysics of Spacecraft and Planetary Bodies, v.20. p.365-878, 1967.

RIEDEL, L. Measurement of thermal diffusivity on foodstuffs rich in water. KaltetechnikKlimatisierung, Karlsruhe, v.21, n.11, p.315-6, 1969.

SWEAT, V.E. Experimental values of thermal conductivities of selected fruits and vegetables. Journal of Food Science, Chicago, n.39, p.1081-3, 1974.

SWEAT, V.E.; HAUGH, C.G. A thermal conductivity probe for small food samples. Transactions of the ASAE, St. Joseph, v.17, n.1, p.56-8, 1974. 